\title{
Diagnóstico de enfermagem "tensão do papel de cuidador": revisão integrativa
}

\author{
Nursing diagnosis "stress in the role of caregiver": an integrative review
}

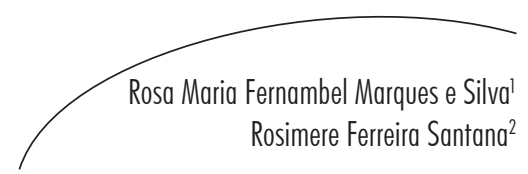

\section{Resumo}

O envelhecimento tornou-se um desafio político-social e, nesse contexto, emerge a determinação correta de sobrecarga dos cuidadores. O objetivo deste trabalho foi identificar e analisar as características definidoras e os fatores relacionados, mediante revisão de literatura integrativa, do diagnóstico de enfermagem "tensão do papel de cuidador", publicado no período 2005-2011. Foram seguidas seis etapas: formulação da questão norteadora; processo de busca na literatura; categorização; avaliação dos estudos; interpretação dos resultados e síntese do conhecimento. Foram pesquisadas as bases LILACS e MEDLINE, e das 66 publicações identificadas, apenas quatro tratavam diretamente do diagnóstico. Evidenciaram-se 24 características definidoras das 34 existentes e 17 fatores relacionados dos 58 referenciados. A tensão do cuidador esteve relacionada aos aspectos físicos, emocionais e socioeconômicos. Assim, o diagnóstico atende às especificidades do cuidador, mas aponta a necessidade de agrupar os indícios clínicos que deem objetividade e clareza necessária para sua identificação na prática de enfermagem.

\section{Abstract}

Aging has become a political and social challenge, and in this context emerges the correct determination of caregiver burden. This work aimed to identify and analyze the defining characteristics and related factors, through integrative literature review, nursing diagnosis "stress in the role of caregiver", published in years 2005-2011. Six steps were followed: formulation of the research question; literature search process; categorization; assessment of studies; interpretation of results and synthesis of knowledge. LILACS and MEDLINE databases were searched, and of 66 publications identified, only four dealt directly with the diagnosis. We found 24 defining characteristics of the existing 34

Palavras-chave: Relações Familiares. Cuidadores. Diagnóstico de Enfermagem. Enfermagem.

Key words: Family Relations. Caregivers. Nursing

Diagnosis. Nursing.

\footnotetext{
Programa de Residência Multiprofissional em Saúde do Idoso, Hospital Universitário Pedro Ernesto. Universidade do Estado do Rio de Janeiro. Rio de Janeiro, RJ, Brasil.

2 Escola de Enfermagem Aurora de Afonso Costa. Universidade Federal Fluminense. Niterói, RJ, Brasil.
}

Correspondência / Correspondence

Rosimere Ferreira Santana

E-mail: rosifesa@gmail.com 
and 17 of the 58 factors referenced. The stress of the caregiver was related to physical, emotional and socioeconomic aspects. Thus, the diagnosis meets the specificity of caregivers, but points to the need to group the clinical signs that give objectivity and clarity to their identification in the nursing practice.

\section{INTRODUÇÃO}

Com as mudanças no perfil demográfico e epidemiológico, o envelhecimento tornou-se um desafio político-social no que diz respeito a estimular a independência e a autonomia dos idosos, e a considerar a importância e sobrecarga dos cuidadores nesse contexto. Trata-se de um fenômeno que necessita de identificação acurada pela enfermagem, com vistas à implantação de um plano terapêutico resolutivo., ${ }^{1,2}$

A sobrecarga vem sendo descrita como frequente devido à sobreposição de tarefas e papéis, ${ }^{3,4}$ acúmulo de atividades, ${ }^{3,5-7}$ deficiências no sistema de saúde, ${ }^{8-10}$ condições de habitação inapropriadas, ${ }^{9,11}$ alto custo do cuidado domiciliar do idoso dependente, ${ }^{8,12,13}$ falta de conhecimento sobre as atividades do cuidado ${ }^{3,5,8,12-14} \mathrm{e}$ isolamento social. ${ }^{3,9,15,16}$ Estes são citados como contribuintes para tensão, estresse e prejuízos à qualidade de vida dos cuidadores.

Cerca de $90 \%$ dos cuidadores são familiares, amigos ou vizinhos, e somente $10 \%$ correspondem aos chamados "cuidadores formais", ou seja, pagos ou contratados. Em sua maioria, são mulheres, esposas e filhas, algo cultural e socialmente estabelecido. Porém, um novo cenário entra em discussão nas pesquisas: a inclusão de idosos cuidando de outros idosos. ${ }^{9,11,17,18}$

$\mathrm{Na}$ prática clínica, para se adotar o diagnóstico de enfermagem proposto pela NANDA-Internacional - tensão do papel do cuidador, definido como dificuldade para desempenhar o papel de cuidador da família ${ }^{19}$ -, observa-se a necessidade de refinamento devido a sua amplitude, ${ }^{20}$ pois é composto por 34 características definidoras e 58 fatores associados e dispostos de forma excedente e inespecífica.

Os enfermeiros, em situações clínicas, ao raciocinarem sobre o que veem, ouvem, cheiram, tocam e sentem o gosto; identificam indícios, que são unidades de dados sensoriais que apontam a conclusão dos diagnósticos. ${ }^{19}$ Daí a necessidade de se investigar quais indícios apontados pela literatura auxiliam na decisão diagnóstica "tensão do papel do cuidador"?

Este estudo objetivou identificar e analisar as características definidoras e os fatores relacionados, mediante revisão de literatura integrativa do diagnóstico de enfermagem "tensão do papel de cuidador".

\section{MÉTODOS}

Trata-se de revisão integrativa de literatura, na qual se procedeu às seis etapas propostas: formulação da questão norteadora; processo de busca na literatura; categorização das publicações; avaliação dos estudos selecionados; interpretação dos resultados dos estudos; e síntese do conhecimento dos artigos analisados. ${ }^{21}$

Estabeleceram-se como questões norteadoras: "qual a definição para tensão do papel do cuidador?" E "quais os elementos/indícios que indicam sua presença?"

Optou-se buscar a literatura em duas bases de dados: Sistema Latino-Americano e do Caribe de Informação em Ciências da Saúde (LILACS) e a Medical Literature Analysis and Retrieval System Online (MEDLINE). Foram definidos como critérios de inclusão: artigos científicos publicados nos idiomas inglês, espanhol e português; 
recorte temporal de 2005 a 2011; relacionados a cuidadores e/ou diagnóstico de enfermagem "tensão do papel do cuidador". Foram excluídos os artigos que tratassem de cuidados prestados por profissionais de enfermagem e dissertações e teses não publicadas.

Elegeram-se como descritores em português, inglês e espanhol: relaçoos familiares, cuidadores e diagnóstico de enfermagem. Em seguida, foram feitas combinações dos descritores "relaçôes familiares and cuidadores". Foram obtidos 36 artigos na LILACS, sendo um excluído por se tratar de dissertação; na MEDLINE, obtiveram-se 30 artigos, dos quais dois foram excluídos por estarem na forma de resumo em anais.

Para as combinações "diagnóstico de enfermagem and cuidadores" na base de dados LILACS, foram obtidos cinco artigos, dos quais dois foram excluídos por se tratar de uma tese e o outro por se repetir. Já na MEDLINE, os sete artigos encontrados aparecem sob a forma de resumo, o que não contempla o critério deste estudo; dois artigos se repetem e nenhum trata do diagnóstico especificamente. Ao total foram analisados 66 artigos, sendo 38 da LILACS e 28 da MEDLINE.

Esses artigos foram lidos e resumidos com o registro de título, autor(es), revista, país de desenvolvimento do estudo e objetivos; foram posteriormente classificados em níveis de evidência registrados no formulário de coleta de dados previamente estabelecido, seguindo-se análise e síntese dos resultados dos estudos.

\section{RESULTADOS}

Quanto ao perfil bibliográfico dos artigos analisados de 2005 a 2011, prevaleceram 36 artigos no idioma português e 30 em inglês. $O$ ano de maior número de publicações abordando o assunto diagnóstico de enfermagem e cuidador foi 2009, com o total de 19 artigos. As categorias profissionais dos autores das publicações são: 62 enfermeiros, 17 psicólogos, seis médicos, três fisioterapeutas e um assistente social.
Aproximadamente $83 \%$ são constituídos por estudos com força de evidência entre os níveis IV, $\mathrm{V}$ e VI, referentes a estudos de baixa evidência clínica. ${ }^{22}$ A maioria (13) é de abordagem qualitativa; quatro são de abordagem quantitativa e três de abordagem quantitativa e qualitativa. Evidenciaram-se dez estudos do tipo descritivo, dez com a utilização de entrevistas semiestruturadas, uma entrevista estruturada, duas observações participantes e um grupo focal. ${ }^{22}$

Das revistas, destaca-se a produção sobre o assunto na Revista Brasileira de Enfermagem (7) e na Revista Latino-Americana de Enfermagem (6). Destas, apenas quatro abordaram a Taxonomia NANDA-I fazendo a associação das consequências físicas e psíquicas quando se trata de um cuidador familiar ao diagnóstico de enfermagem..$^{5,12,23,24}$

Dos quatro estudos que abordaram a Taxonomia NANDA, apenas um estudo ${ }^{24}$ referia-se de fato ao título "tensão", devido ao papel de cuidador entre crianças com câncer; dois eram estudos ${ }^{12}$ de perfis diagnósticos, tratando indiretamente dos diagnósticos de enfermagem de familiares de adultos queimados e dos diagnósticos de enfermagem presentes em cuidadores de idosos com demência $;{ }^{5}$ e um estudo $^{23}$ sobre os determinantes da tensão do cuidador familiar de idosos dependentes.

Emergiram 66 estudos analisados e 17 fatores relacionados, de um universo de 58 fatores existentes propostos pela NANDA (tabela 1).

Dentre as 24 características definidoras evidenciadas em um universo de 34 características existentes, as mais prevalentes foram o estresse, a labilidade emocional aumentada e o afastamento da vida social, conforme representado na tabela 2 .

Os temas recorrentes nos estudos, apresentados na tabela 3 , foram a atuação dos cuidadores, tanto no âmbito hospitalar quanto no domicílio, mais frequentemente sobre a descrição do papel e dos cuidados realizados pelos cuidadores. 
Tabela 1. Fatores relacionados presentes nos 66 artigos analisados levantados nas bases de dados LILACS e MEDLINE entre 2005 e 2011. Niterói-RJ, 2012.

\begin{tabular}{|c|c|}
\hline Fatores relacionados & $\mathrm{n}$ \\
\hline FR 22- Conflito familiar & 9 \\
\hline FR 39- Falta de apoio & 9 \\
\hline FR 15- Problemas físicos & 5 \\
\hline FR 24- Dependência & 5 \\
\hline FR 46- Recursos financeiros insuficientes & 5 \\
\hline FR 57- Isolamento em relação a outras pessoas & 4 \\
\hline FR 01- Alta hospitalar de membros da família com necessidades significativas de cuidado & 3 \\
\hline FR 03- Complexidade das atividades & 3 \\
\hline FR 23- Preocupação com relação aos membros da família & 3 \\
\hline FR 56- Compromissos com vários papéis concomitantes de cuidador & 3 \\
\hline FR 34- Assistência formal insuficiente & 2 \\
\hline FR 44- O cuidador não está pronto do ponto de vista do desenvolvimento & 2 \\
\hline FR 11- Incapacidade de atender às próprias expectativas de outros & 1 \\
\hline FR 14- Problemas cognitivos & 1 \\
\hline FR 16- Problemas psicológicos & 1 \\
\hline FR 27- Instabilidade de saúde do receptor de cuidados & 1 \\
\hline FR 50- Estado mental do idoso que inibe a conversa & 1 \\
\hline
\end{tabular}


Tabela 2. Características definidoras presentes nos 66 artigos analisados levantados nas bases de dados LILACS e MEDLINE entre 2005 e 2011. Niterói-RJ, 2012.

\begin{tabular}{ll}
\hline \multicolumn{1}{c}{ Características definidoras } & $\mathrm{n}$ \\
\hline CD 10 - Estresse & 9 \\
CD 11 - Falta de tempo para satisfazer às necessidades pessoais & 8 \\
CD 14 - Labilidade emocional aumentada & 8 \\
CD 28 - Afastamento da vida social & 6 \\
CD 15 - Nervosismo aumentado & 5 \\
CD 17 - Sensação de depressão & 5 \\
CD 19 - Sono perturbado & 4 \\
CD 01 - Apreensão da capacidade do cuidador para oferecer cuidados & 3 \\
CD 09 - Enfrentamento individual prejudicado & 3 \\
CD 02 - Apreensão em relação à saúde do receptor de cuidados & 2 \\
CD 06 - Dificuldades para realizar as atividades necessárias & 2 \\
CD 22 - Doença cardiovascular & 2 \\
CD 24 - Fadiga & 2 \\
CD 25 - Hipertensão & 2 \\
CD 27 - Problemas gastrointestinais & 2 \\
CD 29 - Baixa produtividade no trabalho & 2 \\
CD 30 - Mudanças nas atividades de lazer & 2 \\
CD 04 - Apreensão quanto aos cuidados que o receptor deve oferecer & 2 \\
CD 08 - Preocupação com a rotina de cuidados & 2 \\
CD 12 - Frustração & 2 \\
CD 13 - Impaciência & 2 \\
CD 16 - Raiva 34 - Pesar quanto ao relacionamento mudado com o cuidado & 1 \\
\hline & 1 \\
\hline
\end{tabular}


Tabela 3. Temas recorrentes nos 66 artigos analisados levantados das bases dados LILACS e MEDLINE entre 2005 e 2011. Niterói-RJ, 2012.

\begin{tabular}{|c|c|}
\hline Temas recorrentes & $\mathrm{n}$ \\
\hline Cuidadores familiares & 33 \\
\hline Idosos & 9 \\
\hline Demência & 8 \\
\hline Crianças & 7 \\
\hline Gênero & 7 \\
\hline Saúde mental & 5 \\
\hline Oncologia & 4 \\
\hline Diagnóstico de enfermagem & 4 \\
\hline Hospital & 3 \\
\hline HIV/Aids & 3 \\
\hline Domicílio & 2 \\
\hline Deficiência auditiva & 2 \\
\hline Dependência química & 2 \\
\hline Acidente vascular encefálico & 2 \\
\hline Disfunção neuromotora & 1 \\
\hline Queimadura & 1 \\
\hline Trauma crânio encefálico & 1 \\
\hline
\end{tabular}

Assim, partindo-se do pressuposto de que o diagnóstico de enfermagem "tensão do papel do cuidador" foi reconhecido em 1992, observase que ainda há poucos estudos relacionados a este diagnóstico (4), em comparação com as publicações referentes a cuidadores - LILACS (729) e MEDLINE (1.106) - e diagnóstico de enfermagem - LILACS (385) e MEDLINE (194) -, no período entre 2005 e 2011.

\section{DISCUSSÃO}

A maioria dos estudos selecionados nesta revisão, em função das fontes de dados pesquisadas, foi produzida no Brasil e indica a relação da temática com o perfil de cuidado domiciliar prestado ao idoso, pois são $53,4 \%$ de lares multigeracionais e $90 \%$ de cuidadores familiares no país. ${ }^{7,915}$ Trata-se de uma atividade que começa a se estender, considerando-se o envelhecimento populacional, e possivelmente por isso o número de publicações tem aumentado em anos recentes.

Tais publicações, no entanto, ainda tratam o fenômeno da sobrecarga/tensão do papel do cuidador em estudos descritivos na busca pela compreensão dos aspectos não só biológicos, mas também psicossociais, os quais tanto interferem na relação com o outro e no bemestar. Este fato aponta a necessidade de compilar os estudos já descritos na identificação correta de "tensão do papel do cuidador", para determinar formas adequadas de intervir e diminuir sua ocorrência. Ou seja, os estudos anteriores colaboram na realização dos futuros 
ensaios clínicos, com análise da efetividade e eficácia das intervenções de saúde.

As publicações indicam atuação do enfermeiro em orientações específicas para familiares de idosos geralmente de baixa renda, em cuidados domiciliares e focados sobretudo no apoio às atividades instrumentais e básicas da vida diária, diminuição do estresse e adaptação do domicílio. ${ }^{25-29}$

O enfoque na utilização do diagnóstico e na linguagem padronizada pode auxiliar o enfermeiro a determinar seu papel profissional. Sobre as características definidoras que conduzem ao raciocínio de tensão do cuidador, estas se iniciam com indícios de ordem psicossocial e, quando não é dada a atenção necessária, progridese para indícios de cunho biológico. $4,5,23,29,30$

O conflito familiar se destaca no julgamento diagnóstico e esteve relacionado à falta de apoio recebido, à dependência e aos recursos financeiros insuficientes. ${ }^{13,15,17} \mathrm{O}$ cuidador se vê sozinho, sobrecarregado e despreparado para as realizações dos cuidados que a longo prazo podem contribuir para problemas de saúde., ${ }^{2,31-33}$

Observa-se que os cuidados realizados se diferenciaram conforme as necessidades de seus familiares. Ocorre a demanda de cuidados para auxílio nas atividades básicas de vida diária (colaboração na higiene pessoal, locomoção e alimentação) ou em atividades instrumentais (ajuda para realizar compras, ir ao médico, administrar recursos financeiros e proporcionar lazer), ${ }^{9,12,23,34-40}$ e o papel do cuidador no processo de recuperação de seu ente. ${ }^{10,34-37}$ Estes assumem uma jornada de mais de oito horas de cuidados sem o apoio de uma segunda pessoa, e ainda têm as atribuições domésticas de limpeza e responsabilidades com demais membros da família e, por vezes, trabalho extra. ${ }^{9,41}$

Essa rotina diária de cuidadoségradativamente citada como geradora de tensão nos cuidadores, com recorrência da falta de conhecimento; a insegurança e o medo de como prosseguirá com os cuidados no domićlilio; da privação da vida social; da interrupção ou adiamento de seus projetos de vida; do desejo, dependência e/ou obrigação de partilhar e acompanhar todos os cuidados; pela falta de recursos financeiros; do conflito familiar na tomada de decisão de quem será o cuidador; na indecisão em institucionalizar ou não; a apreensão quanto ao estado de saúde do ente; a preocupação com os demais membros, com seu emprego e com as novas adaptações requeridas. ${ }^{3,13,24,25}$

A partir disto, pode-se evidenciar que foram recorrentes os seguintes fatores: características definidoras das atividades de cuidado, estado de saúde do cuidador (físico, emocional e socioeconômico), relacionamento entre cuidador e receptor de cuidados; e os relacionados a atividades de cuidado, estado de saúde do cuidador, estado de saúde do receptor de cuidados, processos familiares, recursos; relação entre o cuidador e o receptor de cuidados; e aspectos socioeconômicos. ${ }^{12,19}$ Esses fatores atendem às particularidades experienciadas pelo cuidador na prática e estão contemplados na classificação apresentada, permitindo assim orientar o raciocínio diagnóstico e consequentemente, planejar o cuidado de enfermagem.

O acúmulo de atividades colocadas em prática pelos cuidadores informais/familiar, em sua maioria, esteve relacionado ao gênero. Houve maioria de citações de mulheres classificadas na faixa etária adulta à idosa, havendo apenas quatro estudos da Psicologia ${ }^{36,38-40}$ que refletem a participação do pai enquanto cuidador.

A presença do gênero masculino nos cuidados redefine novos papéis - ou seja, além de sua participação com os recursos financeiros, o homem passa a assumir papel de cuidador que antes era colocado em prática pela mulher. ${ }^{39,41}$ Neste caso observou-se que, independentemente da faixa etária, qualquer fase do ciclo, seja criança, adolescente, adulta ou idosa, pode requerer auxílio de um cuidador. No entanto, isso é mais comum nos idosos, pelo advento das doenças neurodegenerativas e consequentes perdas graduais da cognição. ${ }^{10,13,23}$ 
O aspecto emocional relativo a acompanhar e participar do processo de doença esteve valorizado pelos cuidadores. ${ }^{24} \mathrm{O}$ diagnóstico de enfermagem, neste caso, esteve relacionado à apreensão quanto à recepção de cuidados caso o cuidador adoecesse ou morresse. Isso relevou o sentimento do cuidador, de querer estar próximo e de se entender como a única pessoa capaz de cuidar; e quando a princípio ocorria a ideia de institucionalização do idoso, esta era rejeitada, devido a sentimentos conflitantes de abandono e espera pela morte. ${ }^{8,13}$ Por este motivo, houve citações de saúde fragilizada pela exposição a situações estressoras e também pelo número de hospitalizações do ente, pelas tarefas a supervisionar, pelo agravamento de doenças prévias, poucos recursos socioeconômicos, pelo sentimento de pesar devido às limitações do ente e por seu afastamento da vida social., ${ }^{41,23}$

Os problemas de saúde dos cuidadores mais citados são hipertensão arterial, úlceras, dispepsia, artrite, alterações ponderais, modificações no sistema imunológico, dores lombares, diabetes, hipercolesterolemia, anemia, bursite, varizes, depressão, reumatismo, cefaleia, fratura na perna e pé, alteração intestinal, doença cardíaca e litíase renal. ${ }^{4,9}$ Estes fatores são condicionantes para maior propensão de risco de mortalidade.

Em meio a tamanhas decisões $\mathrm{e}$ responsabilidades, aponta-se para a necessidade de o cuidador encontrar o equilíbrio, para que sua saúde não sofra as consequências de um padrão de sono insatisfatório, cansaço, estresse, sensação de depressão e enfrentamento individual prejudicado. , $^{2,5,9,17,18,23}$

Apontam-se como resolutivas as intervenções para saúde, bem-estar pessoal e apoio social ao cuidador. Para tanto, deve haver a colaboração do enfermeiro, em capacitar tais familiares/ cuidadores na realização dos cuidados, inserindo-os como clientes da enfermagem, além de orientar a rede social quanto à importância do revezamento e divisão das tarefas., ${ }^{1,5,13,18}$

Surgem como limitações do estudo: a dificuldade, na prática clínica, de reconhecer os indícios que levam o cuidador a apresentar o diagnóstico "tensão do papel do cuidador", além do reduzido número de publicações sobre esse diagnóstico.

A revisão integrativa pretende contribuir e incentivar o desenvolvimento de outros estudos, para melhor compreensão do diagnóstico, sua identificação precoce e, consequentemente, melhor qualidade de vida do cuidador.

\section{CONCLUSÃO}

Há um número crescente de estudos relacionados aos diagnósticos de enfermagem, porém poucos trabalham a questão do diagnóstico relacionado ao cuidador, vendo-o como um cliente da enfermagem. A partir da análise da literatura, verifica-se um alto risco para a tensão do papel de cuidador.

Nesta revisão integrativa identificou-se, tanto nos estudos nacionais e internacionais, o sexo feminino como mais propenso a ter o diagnóstico de tensão, o que se correlaciona ao próprio contexto histórico social, que incumbe a mulher como responsável pelo cuidado familiar, e o homem pelo provimento financeiro.

A participação atual da mulher nos cuidados, devido ao envelhecimento populacional, o surgimento das doenças crônicas não transmissíveis e as possíveis limitações e dependência nas atividades instrumentais e de vida diárias se tornaram constantes, sobretudo no domicílio. São atividades que demandam tempo, dificuldades, dedicação e algumas vezes requerem organização familiar, rotinas, alteração de costumes e conciliação de afazeres.

Em sua maioria, são cuidadores informais desprovidos de conhecimentos científicos, que necessitam do auxílio do profissional de saúde e que, em geral, se baseiam empiricamente nos saberes culturais, nas experiências passadas e no que observam e partilham com outros cuidadores.

Ser cuidador, portanto, tem uma representação diferenciada para cada pessoa 
e pode fazer emergir sentimentos positivos e negativos concomitantemente. São sentimentos de retribuição, amor, responsabilidade, satisfação, proximidade; e ora de obrigação, desprazer, sofrimento, comprometimento do seu bem-estar e conformismo. Todo esse cotidiano de excesso ou falta de informações, sobrecarga, cobranças, estresse e adaptações remete gradativamente ao diagnóstico de tensão do papel de cuidador.

As características definidoras mais prevalentes foram as de ordem psicossociais: estresse, falta de tempo para satisfazer às necessidades pessoais e labilidade emocional aumentada. E para os

\section{REFERÊNCIAS}

1. Garbin CAS, Sumida DH, Moimaz SAS, Prado RL, Silva MM. O envelhecimento na perspectiva do cuidador de idosos. Ciênc Saúde Coletiva. 2010;15(6):2941-8.

2. Queiroz ZPV, Lemos NFD, Ramos LR. Fatores potencialmente associados à negligência doméstica entre idosos atendidos em programa de assistência domiciliar. Ciênc Saúde Coletiva. 2010;15(6):2815-24.

3. Azevedo GR, Santos VLCG. Cuida-dor (d)eficiente: as representações sociais de familiares acerca do processo de cuidar. Rev Latinoam Enferm 2006;14(5):770-80.

4. Fernandes MGM, Garcia TR. Atributos da tensão do cuidador familiar de idosos dependentes. Rev Esc Enferm USP 2009;43(4):818-24.

5. Santana RF, Santos I, Caldas CP. Cuidando de idosos com demência: um estudo a partir da prática ambulatorial de enfermagem. Rev Bras Enferm 2005;58(1):44-8.

6. Souza RC, Scatena MCM. Produção de sentidos acerca da família que convive com o doente mental. Rev Latinoam Enferm 2005;13(2):173-9.

7. Fonseca NR, Penna AFG. Perfil do cuidador familiar do paciente com sequela de acidente vascular encefálico. Ciênc Saúde Coletiva 2008;13(4):1175-80.

8. Cesar AM, Santos BRL. Percepção de cuidadores familiares sobre um programa de alta hospitalar. Rev Bras Enferm 2005;58(6):647-52.

9. Oliveira SK. Perfil dos cuidadores de idosos atendidos pelo projeto de assistência interdisciplinar a idosos em nível primário. Ciênc Cuid Saúde 2006;5(2):184-92. fatores relacionados, foram encontrados: conflito familiar, falta de apoio, dependência, recursos financeiros insuficientes e problemas físicos.

Evidenciou-se, assim, um excedente de características definidoras e fatores relacionados, indicando necessidade de "reorganização" e validação clínica do diagnóstico de enfermagem "tensão do papel de cuidador". Quando se definem as características e os fatores relacionados, conforme proposto pela classificação NANDA-I, faz-se a validação deste diagnóstico. Isto significa comprovar que ele é passível de valor prático e teórico para determinada situação clínica, o que dá cientificidade ao conhecimento da Enfermagem.

10. Martins JJ, Do Nascimento ERP, Erdmann AL, Candemil MC, Belaver GM. O cuidado no contexto domiciliar: o discurso de idosos/familiares e profissionais. Rev Enferm UERJ 2009;17(4):556-62.

11. Felix AP, Martins AP, Dyniewicz AM. Capacitação de cuidadores de pacientes em alta hospitalar. Cogitare Enferm 2008;13(1):124-31.

12. Goyatá SLT, Rossi LA, Dalri MCB. Diagnósticos de enfermagem de familiares de pacientes adultos queimados no período próximo à alta hospitalar. Rev Latinoam Enferm 2006;14(1):102-9.

13. Santana RF, Figueiredo NMA, Ferreira MA, Alvim NAT. A formação da mensagem na comunicação entre cuidadores e idosos com demência. Texto Contexto Enferm 2008;17(2):288-96.

14. Machado ALG, Freitas CHA, Jorge MSB. O fazer do cuidador familiar: significados e crenças. Rev Bras Enferm 2007;60(5):530-4.

15. Serna ECH, Sousa RMC. Mudanças nos papéis sociais: uma consequência do trauma crânioencefálico para o cuidador familiar. Rev Latinoam Enferm 2006;14(2):183-9.

16. Santos ECV, Martin D. Cuidadores de pacientes alcoolistas no município de Santos, Brasil. Rev Bras Enferm 2009;62(2):194-9.

17. Rodrigues SLA, Watanabe HAW, Derntl AM. A saúde de idosos que cuidam de idosos. Rev Esc Enferm USP 2006;40(4):493-500.

18. Duca GFD, Thumé E, Hallal PC. Prevalência e fatores associados ao cuidado domiciliar a idosos. Rev Saúde Pública 2011;45(1):113-20. 
19. North American Nursing Diagnosis Association NANDA. Diagnósticos de enfermagem da NANDA: definições e classificação 2009-2011. Porto Alegre: Artmed; 2010.

20. Chaves ECL, Carvalho EC, Rossi L. A validação de diagnósticos de enfermagem: tipos, modelos e componentes validados. Rev Eletr Enferm [Internet] 2008 [acesso em 2 nov 2010];10(2):513-20. Disponível em: http://www.fen.ufg.br/revista/v10/ n2/v10n2a22.htm.

21. Stetler CB, Ritchie JA, Rycroft-Malone J, Schultz AA, Charns MP. Institutionalizing evidence-based practice: an organizational case study using a model of strategic change. Implement Sci 2009;78(4):1-19.

22. Stetler CB, Morsi D, Rucki S, Broughton S, Corrigan B, Fitzgerald J et al. Utilization-focused integrative reviews in a nursing service. Appl Nurs Res 1998;11(4):195-206.

23. Fernandes MGM, Garcia TR. Determinantes da tensão do cuidador familiar de idosos dependentes. Rev Bras Enferm 2009;62(1):57-63.

24. Beck ARM, Lopes MHBM. Tensão devido ao papel de cuidador entre cuidadores de crianças com câncer. Rev Bras Enferm 2007;60(5):513-8.

25. Volpato FS, Santos GRS. Pacientes oncológicos: um olhar sobre as dificuldades vivenciadas pelos familiares cuidadores. Imaginário 2007;13(14):511-44.

26. Marques S, Rodrigues RAP, Kusumota L. Cerebrovascular accident in the aged: changes in family relations. Rev Latinoam Enferm 2006;14(3):102-9.

27. Girondi JBR, Radünz V. A enfermeira como cuidadora do seu familiar com diagnóstico de câncer. Cogitare Enferm 2007;12(2):164-70.

28. Carvalho AMA Cavalcanti VRS; De Almeida MA; Bastos ACS. Mulheres e cuidado: bases psicobiológicas ou arbitrariedade cultural? Paidéia (Ribeirão Preto) 2008;18(41):431-44.

29. Botti ML, Leite GB, Do Prado MF, Waidman MAP, Marcon SS. Convivência e percepção do cuidado familiar ao portador de HIV/AIDS. Rev Enferm UERJ 2009;17(3):400-5.

30. Barroso SM, Bandeira M, Nascimento E. Fatores preditores da sobrecarga subjetiva de familiares de pacientes psiquiátricos atendidos na rede pública de Belo Horizonte, Brasil. Cad Saúde Pública 2009;25(9):1957-68.

Recebido: 15/4/2013

Revisado: 23/4/2014

Aprovado: 17/6/2014
31. Awadalla AW, Ohaeri JU, Gholoum A, Khalid AOA, Hamad HMA, Jacob A. Factors associated with quality of life of outpatients with breast cancer and gynecologic cancers and their family caregivers: a controlled study. BMC Cancer 2007;102(7):1-7.

32. Hughes ME, Waite L, LaPierre T, Luo Y. All in the family: the impact of caring for grandchildren on grandparents' health. J Gerontol Ser B Psychol Sci Soc Sci 2007;62(2):108-19.

33. Miliorini JP, Fernandes MV, Decesaro MN, Marcon SS. A família no contexto hospitalar: apreendendo os anseios e expectativas relacionadas com doença crônica. Rev Rene 2008;9(3):81-91.

34. Li-Chueh W, Hsiu-Li H, Yi-Wen W, Chia-Ling C, Cheng-Hui T, Wei-Chen L. Primary caregiver stress in caring for a living-related liver transplantation recipient during the postoperative stage. J Adv Nurs 2011;67(8):1749-57.

35. Kim SS, Reed PG, Hayward RD , Kang Y, Koenig HG . Spirituality and psychological well-being: testing a theory of family interdependence among family caregivers and their elders. Rev Nurs Health 2011;34(2):103-15.

36. Machado WCA, Scramin AP. (In)dependência funcional na dependente relação de homens tetraplégicos com seus (in)substituíveis pais / cuidadores. Rev Esc Enferm USP 2010;44(1):53-60.

37. Cachioni M, Lima-Silva TB, Ordonez TN, GaloTiago J, Alves AR, Suzuki MY, Da Silva DV, Falcão DVS. Pacientes idosos com demência de Alzheimer e suas relações familiares: perspectivas do cuidador. Dement Neuropsychol 2011;5(2):114-22.

38. Tronchin DMR, Tsunechiro MA. Cuidar e conviver com o filho prematuro: a experiência do pai, que refletem a participação do pai enquanto cuidador de seu filho. Rev Latinoam Enferm 2006;14(1):93-101.

39. Crepaldi MA, Andreani G, Hammes PS, Ristof CD, De Abreu SR. A participação do pai nos cuidados da criança, segundo a concepção das mães. Psicol Estud 2006;11(3):579-87.

40. Bolsoni-Silva TB, Paiva MM, Barbosa CG. Problemas de comportamentos de crianças / adolescentes e dificuldades de pais / cuidadores: um estudo de caracterização. Psicol Clín 2009;21(1):169-84.

41. Pavarini SCI, Tonon FL, Silva JMC, De Mendiondo $\mathrm{Mz}$, Barham EJ, Filizola CLSAl. Quem irá empurrar minha cadeira de rodas? A escolha do cuidador familiar do idoso. Rev Eletrônica Enferm [Internet] 2006 [acesso em 29 nov 2010];8(3):326-35. Disponível em: http://www.revistas.ufg.br/index.php/fen/ article/view/7071/5002. 